See discussions, stats, and author profiles for this publication at: https://www.researchgate.net/publication/266618886

\title{
Performance analysis of bee-hive routing in multi-radio networks
}

Conference Paper · February 2014

Dol: 10.1109//AdCC.2014.6779349

CITATIONS

2

8 authors, including:

\section{Kiran $\mathrm{K}$}

University Visvesvaraya College of Engineering

17 PUBLICATIONS 33 CITATIONS

SEE PROFILE

P. Deepa Shenoy

University Visvesvaraya College of Engineering

178 PUBLICATIONS 1,297 CITATIONS

SEE PROFILE

Some of the authors of this publication are also working on these related projects:

Web caching and prefetching View project

Discovery of loT Objects View project
READS

63

Abhishek Alfred Singh

Nokia

8 PUBLICATIONS 20 CITATIONS

SEE PROFILE

Venugopal K R

University Visvesvaraya College of Engineering

925 PUBLICATIONS 3,707 CITATIONS

SEE PROFILE 


\section{Performance Analysis of Bee-Hive Routing in Multi-radio Networks}

\author{
Kiran K, \\ Assistant Professor \\ Shivapriya T, Abhishek Alfred Singh, Nazima Begum, Ramya R, \\ Students \\ $P$ Deepa Shenoy, Venugopal $K R$ \\ Professors \\ Department of Computer Science and Engineering, \\ University Visvesvaraya College of Engineering \\ Bangalore, India
}

\author{
Lalit M Patnaik \\ Honorary Professor, \\ Indian Institute of Science \\ Bangalore, India
}

\begin{abstract}
In recent years, wireless communication technology has reduced the distance between people and has hence become a significant part of our lives. Two such technologies are WiFi(IEEE 802.11) and WiMAX(IEEE 802.16) where the latter is a long range system covering many kilometers, whereas former is a synonym for WLAN providing a coverage of only short ra nges. This work describes the implementation of a framework in which a multi-hop, ad-hoc network is deployed with hybrid nodes to enhance network throughput. The data traffic received is split between the WiFi and WiMAX radios on the basis of the split coefficient value statically. The routing algorithm being implemented in this paper is the be e-hive algorithm. Bee-hive algorithm is a multi-path routing algorithm inspired by the social behavior of swarms of bees. It is dynamic, robust and flexible yet s imple algorithm which can prove helpful for optimal management of available network resources. In this paper, we have split data traffic over two radio channels for achieving enhanced performance and reduced delay.
\end{abstract}

Keywords-Ad-hoc Wireless Networks, Bee-Hive Rou ting, Multi-Radio Nodes, Traffic Splitting, Swarm Intelligence

\section{INTRODUCTION}

The need for fast data connection has led to the evolution of wireless communication networks in recent years. WiMAX (IEEE 802.16) and WiFi(IEEE 802.11) are two such technologies which have aided in achieving the goal of faster internet access among other competing technologies. WiMAX (Worldwide Interoperability for Microwave Access) is capable of providing coverage to greater distances, where as WiFi's(Wireless Fidelity) coverage area is limited to shorter ranges.

Although WiFi and WiMAX are designed to fulfil different requirements, they are complementary to each other. To exploit the best features of both the technologies, we have combined and put them up in a single node. In our work we have used hybrid nodes, each of them equipped with a wifi radio and a WiMAX radio, and the incoming data traffic is split between these two radios. Here a traffic splitting coefficient has been defined which denotes the percentage of data traffic split over the two channels.

Selection of appropriate routing algorithm plays a crucial role in the performance of the network. In this work we have implemented slightly modified version of bee-hive routing algorithm to route the packets to meet our requirements. Beehive algorithm is basically influenced by the foraging behaviour of bees. It is one of the stepping stones towards the intelligent networks that optimally manage the available resources.
Certain problems cannot be tackled by merely using the traditional algorithms, but they can be demystified using the natural mechanisms. Swarm intelligence inspired by the behavior of the social insects, is a natural engineering approach, where goals and challenges of a real world system are discovered before the actual implementation of the solution. This artificial intelligence technique focuses on collective behavior in the systems which are populated by simple agents. The behavior of these agents is controlled by the local interactions with one another and their environment that often results in a global pattern.

We can find systems of this kind in nature. Some of the popular and successful algorithms associated with swarm intelligence are namely Ant Colony Optimization, Birds Flocking and Foraging algorithms. The observation which is the seed for these algorithms is that if we visualize the group of natural organisms like ants, bees, bacteria itself as an individual, then the swarm would be more intelligent than any of its members taken individually. Such a scenario in which a collection of agents exhibit behavior that is not possible for the individuals is especially observed in bio-sciences. For example ants finding shortest distance path to reach their nest or honey bees locating the best nectar sources closer to their hives.

The two basic principles behind swarm intelligence are self-organization and stigmergy. Self-organization is a set of dynamical mechanisms where the structures appear at the global level of a system from interactions of its lower-level components[3]. Stigmergy is nothing but an indirect communication via signs or cues given by an individual to convey some useful information to rest of the members of the swarm who encounter them. This forms the basis of the cooperation among the social insects.

Bee-hive algorithm is one such algorithm being used for the purpose of routing in this paper. The underlying idea behind this algorithm is the information exchanging skills of the honey bees. Here the method adapted by the bees to search and exploit good food sources is implemented in a packet switching network for the purpose of finding suitable paths to the destination. This algorithm makes sure that the local routing tables of all nodes along the path are updated with the most recent information.

This paper is structured into six sections. Section II gives an overview of the works done earlier in this context. Section III is devoted to the discussion of the underlying concepts for this work followed by the problem statement in section IV. 
Section $\mathrm{V}$ deals with the implementation of modified beehive algorithm for traffic splitting of data in a multi-hop, multi-radio network. Finally, we conclude our work in Section VI.

\section{RELATED WORK}

This work mainly focuses on two concepts- multi-radio networks and bee-hive routing algorithm. In this section we throw light on the related works done in these areas. Literature [1] deals with a new routing mechanism called Proactive Route Maintenance (PRM) in which routing information is spread widely along active routes and advertised only by active nodes that forward data packets. PRM achieves better load distribution and higher route reliability by forwarding data on several optimal paths[1].

Paper [2] proposes a framework that makes the simulation of wireless architecture possible by exploiting the radio hierarchies for power-efficient neighbour and service discovery and connection setup. In the scenario considered, each node is equipped with a Lower-Power (LP) and a HighPower (HP) radio where the former broadcasts beacons at regular intervals and remain always turned on and latter gets switched on only when a node receives a beacon from its neighbour and gets suspended automatically after transmission[2].

In literature[3], a review on swarm intelligence has been presented. A novel adaptive routing technique called AntNet has been developed in [4], which is based on mobile agents. This routing technique is currently used in packet switching networks and results in better throughput compared to other commercial algorithms[4]. The idea of Distributed Genetic Algorithms (DGA) has been put forth in literature[5]. In this algorithm the overhead of maintaining large number of entries in the routing table is reduced by traversing the agents in a particular order called chromosome consisting of six nodes [5].

Paper [6] Emphasizes on enhancement of throughput in a multi-radio network by splitting of data traffic over the two radio channels using AODV algorithm. The work done in [7] is aimed at analyzing the effect of traffic splitting in a multiradio network. Literature [8] shows that the network-wise performance evaluation method provides good accuracy in estimation of end-to-end delay and delay variance of a network through numerical experiments. Literature [9] deals with the integration of WiFi and WiMAX technologies in order to improve network throughput.

\section{BACKGROUND}

Congestion is one of the major contributors to the deterioration of network performance. Congestion is a state which arises when the network is overloaded with huge number of packets which results in loss of packets and transmission delay. Consequently the performance plummets as the network is exploited beyond its potential. Therefore, the most effective way to control congestion would be reducing the load on the network.

In our work the aim of the load reduction is accomplished by using multi-radio nodes in the network. Here we are using hybrid nodes in which WiMAX and WiFi radios are simultaneously used for transmission and reception of packets. The hybrid node splits the data traffic over these two radio channels according to the fixed value of the split coefficient. Traffic splitting takes place only at the source node.
The rest of this section is dedicated to the detailed discussion of the bee-hive routing algorithm. It is a bioinspired algorithm based on the concept of swarm intelligence, which is all about achieving a definite goal by working together in a system. The characteristics of swarm intelligence that leads to performance optimization and robustness are indirect interactions and distributed task allocation among the individuals in a decentralized and selforganized system.

In this algorithm, bee agents travel through network regions called foraging zones and communicate information about the food sources to their fellow foragers through a waggle dance inside the bee hive on the dance floor[5]. Foraging is the behavior through which the swarm explores and exploits rich nectar sources that are close to its hive.

The term waggle dance refers to the particular figure eight dance of the honey bees. Through this dance the bees communicate the distance, direction and quality of the food source to the fellow honey bees and try to recruit as many foragers as possible for exploiting that site fully. Longer the duration of the dance the more abundant is the source. In this way the swarm of bees follows smart resource allocation by recruiting more number of bees to rich nectar sites and gradually reduces the duration of the dance as the source becomes depleted.

\section{Problem STATEMENT}

In this work we are developing a multi-hop, multi-radio network using mobile nodes in which data traffic is split statically. Here our main intention is to analyze the performance of this network using bee-hive routing algorithm. As mentioned earlier we have defined a parameter split coefficient which denotes the percentage of data traffic split over the two radio channels. We are trying to study the effect of this parameter on the throughput and the delay of the network.

\section{IMPLEMENTATION AND ANALYSIS}

The bee algorithm is explained in [5]. Few modifications are added to the beehive algorithm in order to work in a multi-radio network. The modified beehive algorithm is explained below. The routing tables needed are:

- IFZ (neighbor_addr, propogation_delay, packet_list)

- RS (dest, nexthop, packet_list)

- WT (dest, num_try, event_id, found, prenodeList)

IFZ stores neighbor's address and propagation delay to it and list of packets associated with each neighbor address. RS stores destination to reach and neighbor address that will be able to send packet in proper direction is stored in nexthop field and packet list containing packets that are to be sent to that destination. WT stores the details of destination for which enquiry bee is launched and num_try tells the num of times bee launched event id this stores the id of timer expiry, prenodeList is the list of nodes that are waiting to be informed about the path to that destination. In our implementation few modifications are added in order to work in a multi-radio network initialized short limit $=3$ and long limit=7. THRESHOLD is the distance range set, based on WiFi or WiMAX part when a data packet with unknown destination is generated; an enquiry bee is launched to find the path to destination and starts a short timer for it. Any node that receives the enquiry bee will check its routing tables for the destination. If found, generates and sends 
reply to the node that sent enquiry, else forwards the bee to its neighbors. A node on receiving reply will update its routing table and forward it to all nodes in the prenodelist (all nodes which sent enquiry bee and waiting for reply). When short timer expires, node checks whether path exist, if not generate a long enquiry bee and send to neighbors and start a long timer. When long timer expires, node checks if a valid path exist, if not destroy packets and update routing table. The pseudo code of the Modified BeeHive algorithm is given in Table I.

Table I. Pseudo code of the Modified BeeHive Algorithm

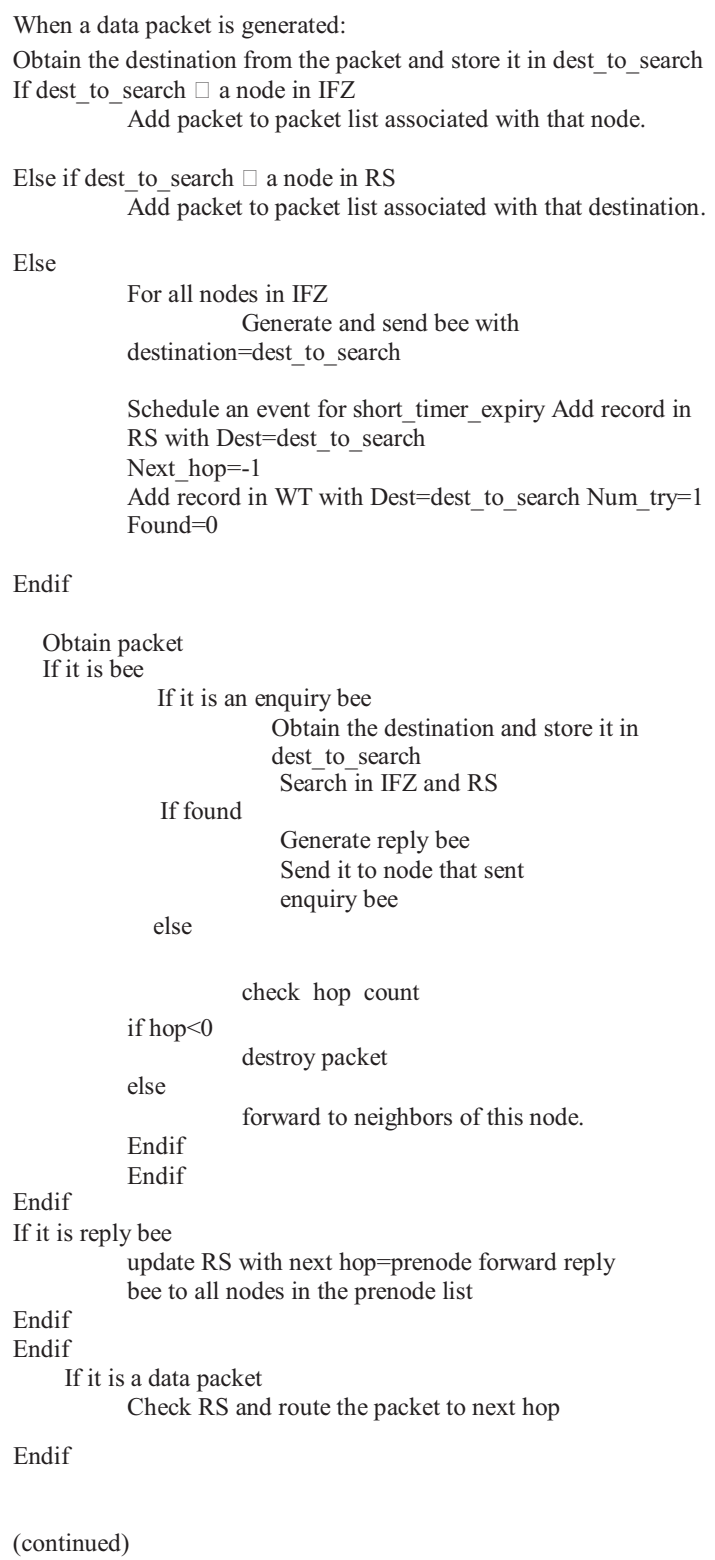


TABLE III. SIMULATION RUN II METRICS

The Network layer implements the Modified BeeHive Routing algorithm. The Modified BeeHive Routing algorithm was described in Table I.

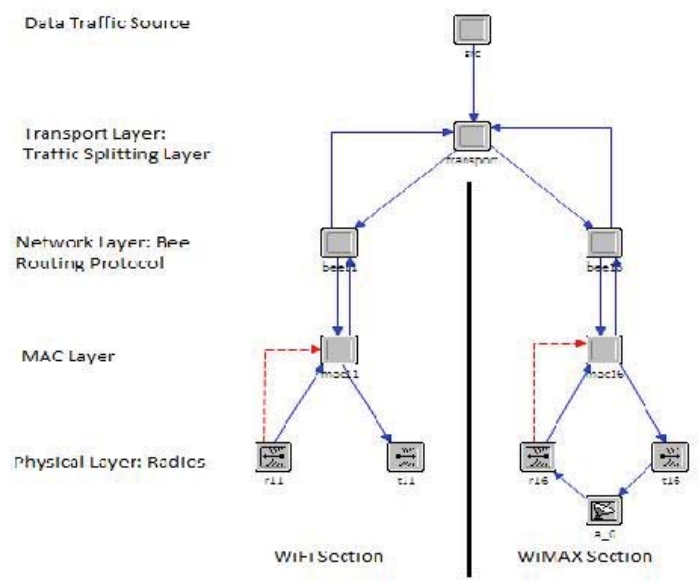

Fig. 3. Hybrid Node Layered Design

The Traffic Splitting layer is implemented just above the network layer. Its main task is to split data traffic from the data source to the WiMAX or WiFi vertical sections in the node as shown in Fig.3. Traffic splitting is performed based on a simple algorithm of transmitting a percentage of traffic through the WiMAX section and the rest over the WiFi Section.

The algorithm for splitting data traffic is discussed in [6]. In the algorithm the traffic is split as a ratio [7] signifying the percentage of data traffic routed over WiMAX or WiFi Radio channels. The traffic split ratios are adjusted statically for each run of the simulation. The graphs are plotted for Delay and Throughput varying with time for various values of split ratios. Split ratios or split coefficients here are selected as an integer value between 0 and 10 inclusive.

A split ratio value of 4 implies that $40 \%$ of data traffic is routed over WiMAX and the rest is routed over $\mathrm{WiFi}$. The simulation is executed for 600 seconds for different values of Split ratios varying between 0 and 10 inclusive. It must be clear that a Split ratio of 0 implies that the data is completely transmitted over the WiMAX channel and a split ratio of 10 implies data traffic transmitted completely over the WiFi Channel.

TABLE II. SimULATION RUN I METRICS

\begin{tabular}{|c|c|c|}
\hline \multirow{2}{*}{ Radios } & \multicolumn{2}{|c|}{ Parameters for Simulation Run I } \\
\cline { 2 - 3 } & Raw Data Rate & Radio Range \\
\hline WiMAX & $8 \mathrm{Mbps}$ & $2000 \mathrm{~m}$ \\
\hline WiFi & $54 \mathrm{Mbps}$ & $250 \mathrm{~m}$ \\
\hline
\end{tabular}

\begin{tabular}{|c|c|c|}
\hline \multirow{2}{*}{ Radios } & \multicolumn{2}{|c|}{ Parameters for Simulation Run II } \\
\cline { 2 - 3 } & Raw Data Rate & Radio Range \\
\hline WiMAX & $54 \mathrm{Mbps}$ & $2000 \mathrm{~m}$ \\
\hline $\mathrm{WiFi}$ & $54 \mathrm{Mbps}$ & $250 \mathrm{~m}$ \\
\hline
\end{tabular}

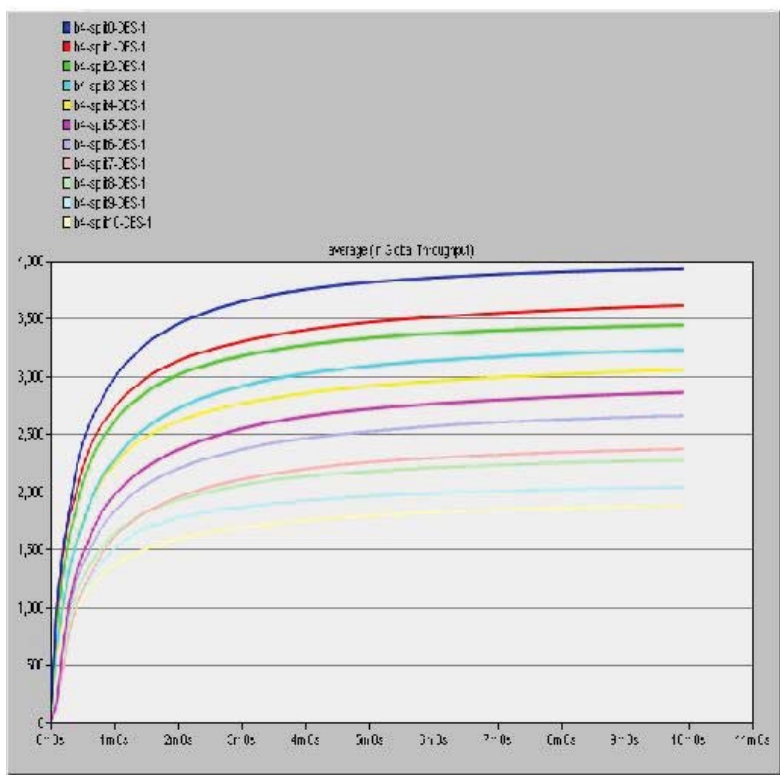

Fig 4. Throughput Graph for Run I for different values of Split Ratio.

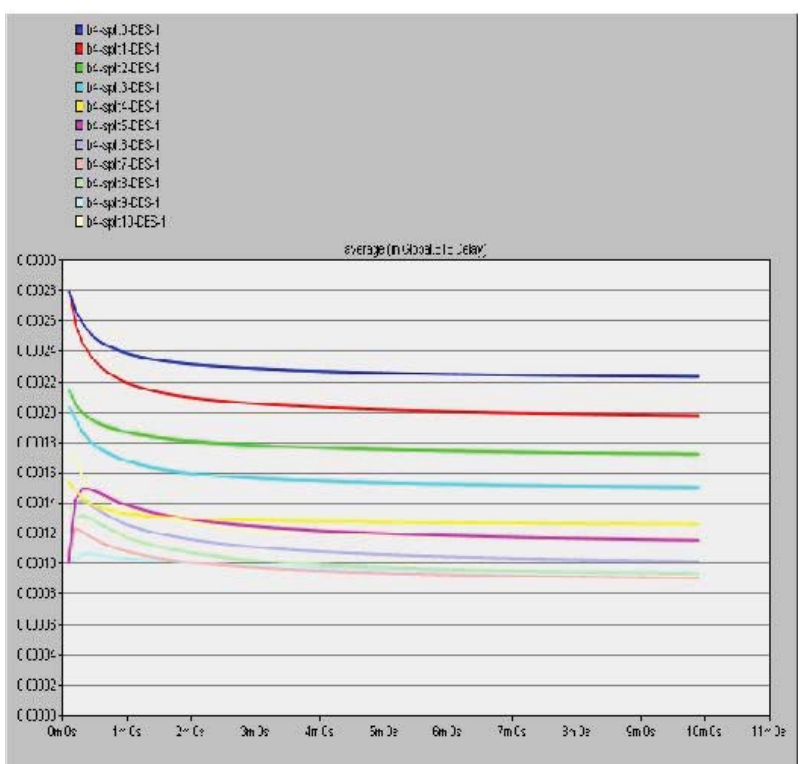

Fig 5. Delay Graph for Run I for different values of Split Ratio 


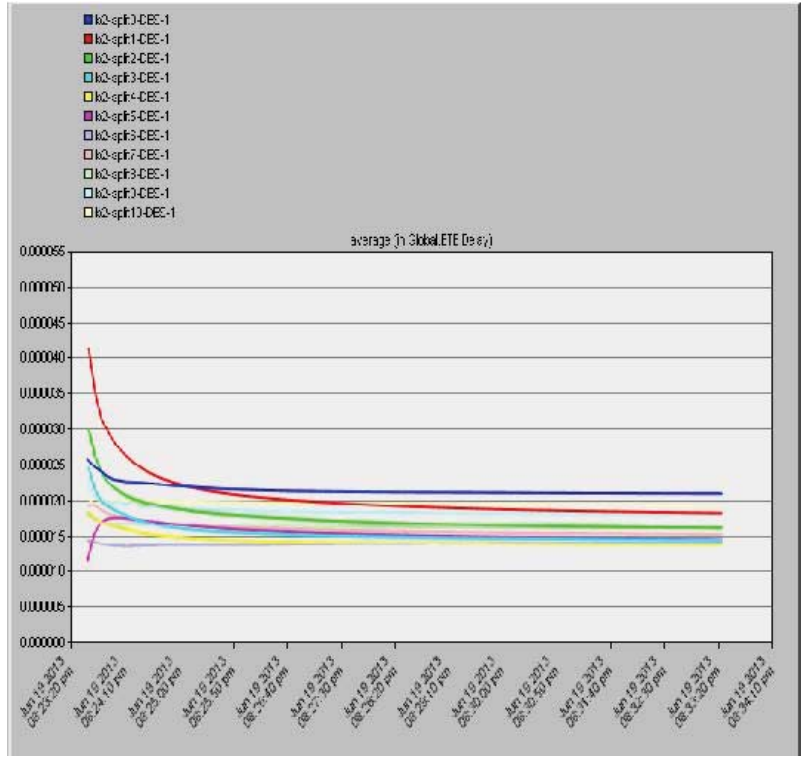

Fig 6. Delay Graph for Run II for different values of Split Ratio.

Table II and III, show the simulation metrics used during the simulation of the network shown in Fig. 2. Graphs are plotted for Throughput and Delay for overall data transmitted in the network. These graphs are shown in Fig. 4, Fig. 5, Fig.6 and Fig. 7.

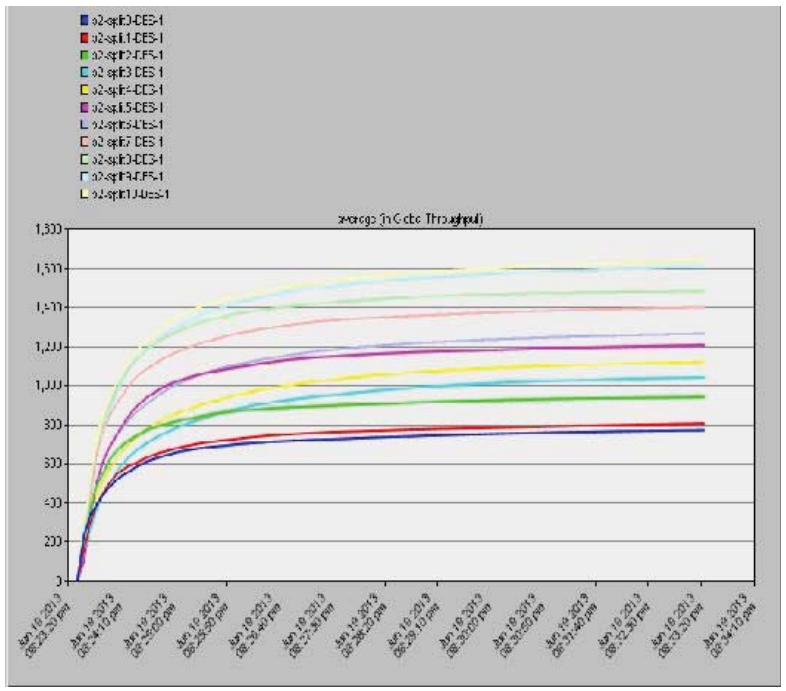

Fig 7. Throughput Graph for Run II for different values of Split Ratio.

The graphs in Fig. 4 and Fig. 5 are plotted for the values of range and data rate shown in Table II. In Fig. 4, Throughput is plotted for a simulation run time of 600 seconds for different values of Split Ratios. Fig. 5 shows End-to-End delay for packet transmission p lot te d for different values of split ratios.

The graphs in Fig. 6 and Fig. 7 are plotted for values of range and raw data rates shown in Table III. The graph in Fig. 6 shows the variation of delay with different values of split ratios. Fig. 7 shows variation of throughput with time for different values of split ratios.

It can be seen from the graphs that the delay is significantly reduced for a split ratio of around the 6 to 8 mark. The throughput also is seen to increase in this range.
This shows that data routed over multiple channels using BeeHive routing reduces the overall delay in transmission as well as improves the throughput.

\section{CONCLUSION AND Future WORK}

In this paper, we have discussed a method of applying BeeHive Routing protocol in a Traffic Splitting scenario over Multi-hop, Multi-radio channels. Our simulations used the raw data rate and approximate range of the WiMAX and WiFi radios. It is seen from simulation results that the delay and throughput of the Multi-hop, Multi-radio network is improved by splitting data traffic over two different radio channels. In the present work we have focused on arranging static values of split ratios. These are generally not seen in field conditions and our main aim of future research work will be to look at optimization techniques to calculate split ratios based on various network parameters such as congestion, architecture, QOS guarantees, etc.

\section{REFERENCES}

[1] Fei Dai and Jie Wu. "A highly reliable multi-path routing scheme for ad hoc wireless networks", The International Journal of Parallel, Emergent and Distributed Systems, Vol. 00, No. 0, 2005, 1-15.

[2] Ólafur Helgason and Sylvia T. Kouyoumdjieva. "Enabling Multiple Controllable Radios in OMNeT++ Nodes", in Proceedings of ICST Conference on Simulation Tools and Techniques (SIMUTools'11), OMNeT++ workshop, Barcelona, Spain, March 2011.

[3] E. Bonabeau, M. Dorigo, and G. Theraulaz. Swarm Intelligence: From Natural to artificial Systems. Oxford University Press, 1999.

[4] B.Bar'an and R.Sosa. "A new approach for Antnet Routing," Proceedings of the Ninth International Conference on Computer, Communications and Networks, 2000.

[5] Horst F. Wedde, Muddassar Farooq, and Yue Zhang. "BeeHive:An effiecient routing algorithm inspired by Bee behavior," SpringerVerlag, pp-83-94, 2004

[6] Kiran K, Abhishek Alfred Singh, Yadunandan S, P Deepa Shenoy, Venugopal K R and L.M.Patnaik, "Throughput Enhancement by Traffic Splitting over an Ad-Hoc Network with Hybrid Radio Devices," Proceedings of IEEE Tencon Spring 2013, pp 401-5.

[7] Kiran K, Abhishek Alfred Singh, P Deepa Shenoy, Venugopal K R and L.M.Patnaik, "Analysis of Traffic Splitting over a Multi-hop Network with Hybrid WiMAX and WiFi Nodes", IEEE Conference on Parallel Grid and Distributed Computing, Simla, 2012.

[8] Huei-Wen Feng and Cheng Ching peng, "Traffic Splitting and ts Applications to Network-Wise Performance Analysis," Symposium n on Performance Evaluation of Computer and Telecommunication Systesm, pp 494-501, 2003.

[9] Ghazisaidi N,Kassaei H, and Bohlooli M.S.,'Integration of WiFi and WiMAX Networks," International Conference on Advances in Mesh Networks, pp 1-6, June 2009. 